S. Kwok, M. Dopita, and R. Sutherland, eds.

\title{
The Effects of Transition Time on the Populations of Post-AGB Stars, and on the Nebular Evolution.
}

\author{
Eva Villaver, Letizia Stanghellini \\ Space Telescope Science Institute, 3700 San Martin Drive, Baltimore, \\ $M D$ 21218, USA \\ Arturo Manchado \\ Instituto de Astrofísica de Canarias, c/ Via Láctea s/n $38200 \mathrm{La}$ \\ Laguna (Tenerife), Spain \\ Guillermo García-Segura \\ Instituto de Astronomía-Universidad Nacional Autónoma de México, \\ Apartado Postal 877, Ensenada, 22830 Baja California, México \\ Alvio Renzini \\ European Southern Observatory, Karl-Schwarzschild-Str. 2, 85748 \\ Garching, Germany
}

\begin{abstract}
We show how the different assumptions on the transition time (the time lag between the superwind quenching at the AGB and the illumination of the Planetary Nebula $[\mathrm{PN}]$ ) reflect into very different theoretical outcomes, both in the characteristics of the stellar remnants, and in the evolution of the nebular shells.

We use a Monte Carlo simulation of post-AGB stars with a set of assumptions on the transition time, to show the effect on the resulting location of the stars on the HR diagram, and on the derived core mass distributions.

We have also performed numerical simulations of the PN formation process, and investigated the effects of the transition time on the resulting PN structure. We found that the transition time determines not only the size of the PN shell, but also its dynamical evolution.

We show the important implications that the transition time has on the observable parameters during the PN stage.
\end{abstract}

\section{The effect of the transition time on the stellar populations}

Stellar evolution models skip the transition phase, with the consequence that the post-AGB evolutionary tracks start with ad hoc models. For this reason, it is hard to compare the stellar evolution models with the observational data, in that it is like using two unsynchronized clocks. 
The transition time depends essentially on $M_{\mathrm{e}}^{\mathrm{R}}$, the residual envelope mass that is left on the star after the nebular ejection. $M_{\mathrm{e}}^{\mathrm{R}}$ is not well constrained by the stellar evolution models, nor by the observations. The residual envelope mass determines the wind time scale and the nuclear time scale, a combination of whose gives the transition time, assuming that the stellar remnant does not evolve on a thermal time scale (in this last case, the transition time is virtually zero, see Stanghellini \& Renzini 2000).

In order to show the effects of the transition time we use a Monte Carlo simulation of post-AGB stars with same IMF, same initial mass-final mass relation, same number of initial objects, and different transition times. As a result, we obtain very different distributions on the $\log T_{\text {eff }}-\log \mathrm{L}$ plane. We find that the differences are even more noticeable in the core mass distribution derived from these synthetic populations.

\section{The effect of the transition time on the PN evolution}

We have performed numerical simulations of the PN formation process including the wind evolution during the AGB. The effect of the transition time is explored in the models in two ways: first, we artificially set the transition time to zero, and second, we use the prescription given by Vassiliadis \& Wood (1994). We found that the transition phase has a strong effect on the shell evolution. When the transition occurs instantaneously, both the ionization front (IF) and the fast stellar wind encounter higher densities in the inner parts of the evolving nebula. As a consequence, the size of the main PN shell is smaller, and the expansion velocities are slower during the early $\mathrm{PN}$ stages when compared to the non-zero transition time models. Moreover, due to dependence of the IF propagation velocity with density, adopting zero transition time the nebula remains ionization bounded for a long time. Therefore, the central star is very hot when the nebula becomes density bounded. The models that have non-zero transition times have lower densities in the inner parts, and are pushed by the fast wind more efficiently. We also found that the stellar mass and the radius reached by the inner shell for the models with non-zero transition times are directly related.

This study is the background for a wider analysis on the effects of theoretical assumptions in the phases immediately following the nebular ejection. We will apply the prescriptions by Stanghellini \& Renzini (2000) to the hydrodynamical models, to finely tune the transition time effects to the observed PNe.

\section{References}

Stanghellini, L. \& Renzini, A. 2000, ApJ, 542, 308

Vassiliadis, E. \& Wood, P. 1994, ApJS, 92, 125 